\title{
Multiple huge abscesses of sacroiliac joint and hip: tuberculosis or pyogenic infection? Treatment and literature review
}

\section{Yujia Li}

Second Xiangya Hospital

Jiepeng Xiong

Changsha central hospital

\section{Yun Gu}

Changsha central hospital

Min Yu ( $\nabla$ docyummy@126.com )

Changsha central hospital https://orcid.org/0000-0001-9683-9182

\section{Ke Chou}

Changsha central hospital

\section{Case report}

Keywords: infection, sacroiliac joint, abscess

Posted Date: March 4th, 2021

DOI: https://doi.org/10.21203/rs.3.rs-270634/v1

License: (c) (1) This work is licensed under a Creative Commons Attribution 4.0 International License. Read Full License 


\section{Abstract}

Background: Sacroiliac joint infection is uncommon in clinical. It's initial symptoms are usually nonspecific, often confused with tuberculosis, septic arthritis of the hip, osteitis of the ilium and lumbar disc herniation which make it difficult to early diagnosis. There is few report about the sacroiliac joint infection, especially with multiple huge abscesses.

Case presentation: A 29 years old male who developed pain of the right buttock, and firstly was diagnosed as lumbar disc herniation, then sacroiliac joint tuberculosis. The therapeutic effect was not satisfactory. We performed surgical debridement for him and get the clear diagnosis which is staphylococcus aureus infection of sacroiliac joint. After thoroughly debrided, the symptoms disappeared with adequate and regular antibiotic therapy.

Conclusions: For sacroiliac joint infection, clinical suspicion is low, the condition is uncommon, and the general incidence is unknown. It's presentation is diverse. So when encountered similar situations, the sacroiliac joint infection should be considered, and the pathogen should be identified, in order to treat it correctly as soon as possible.

\section{Background}

Sacroiliac joint infection is not common, especially with multiple huge abscess. The clinical symptoms include low back pain originating in the sacroiliac joint, mainly occured in the gluteal region, meanwhile is often radiate to the lower and upper lumbar region, groin, abdomen, and/or lower limb, which are not typical and very similar to the symptoms of lumbar disc herniation, so it is often confused which make sacroiliac joint infection difficult to early diagnose. The radiological features of the sacroiliac joint infection are localized bone destruction with soft tissue abnormality even with abscess, which is hard to distinguish from tuberculosis. We present a case of sacroiliac joint infection with multiple huge abscesses.

\section{Case Presentation}

A 29 years old male was admitted to hospital for complaining of pain of the right buttock for more than 2 weeks. No abnormality found in physical examination of the spine and left hip, the ROM of the spine and the hip is normal, straight-leg raising test is negative, the gait is normal. No abnormality was found in radiology(figure a). Consider the above, the doctor suggest that she reduce daily movement, have a rest, lie down and take some physiotherapy, include traction, magnetic thermotherapy and wear waistline. The symptoms did not improve significantly and 1 week later he gradually showed limited walking activity, difficulty getting up, fever and chills. He was obviously chilly at night and the highest body temperature was 40 degrees. The laboratory examination: blood routine examination: white blood cell $14.34 \times 10^{9} / \mathrm{L}$; neutrophil count $12.0 \times 10^{9} / \mathrm{L}$; neutrophil ratio $84.00 \%$; ESR:45mm/h; CRP:19.60mg/L, which suggesting infection. Then he was referred to orthopaedic surgeon, who requested $\mathrm{CT}$ (figure $b$ ) and magnetic 
resonance image(figure $\mathrm{c}$ ) of the pelvis and hip. CT show narrowing of sacroiliac joint space, cortical discontinuity of articular face and osteosclerosis, Multiple soft tissue infections (abscess formation) in right iliolumbar and hip, considering infectious lesions. MRI manifest that abscess formation and bone marrow edema on the right side of sacral vertebra, infection was considered. SPECT show increased bone metabolism in right sacroiliac joint which indicates the tuberculosis of bone and joint. So he received anti tuberculosis therapy. At first, the effect was fair, he feel the symptom was relieved slightly, but still feel low back pain, especially when sitting. Until the result show that tuberculosis antibody IgG+lgM was negative; TB infection T cell detection: negative; PPD(-), Xpert MTB/RIF mycobacterium tuberculosis and rifampicin resistance monitoring: mycobacterium tuberculosis complex DNA negative. It is suspected that the diagnosis is not tuberculosis. Then the pus was collected by puncture, and the culture result shows staphylococcus aureus, moxifloxacin sensitive, and acid fast bacilli smear negative. After anti infection treatment with moxifloxacin, operation was arranged. Surgery was performed under general anesthesia, the infected tissue and abscess were thoroughly debrided(figure d) with adequate drainage and anti infective therapy. 1 week later when the infection was controlled satisfactory, the wound was debrided again and repaired in the second stage. The patient's symptoms disappeared completely and did not recur.

For this case, when the patient come to us, the symptoms have worsened and abscess have formed, plus the laboratory evidence, the infection of sacroiliac joint was definite, but the pathogen of infection is undefinite. We are not sure whether it is pyogenic infection or tuberculosis infection with abscess formation. They have similar symptoms and imaging findings and easy to be misdiagnosed. Clinically, the diagnosis may be obscured by hip pain and poorly localising signs of infection with or without fever. So it is frequently associated with diagnostic delay. But treatment should be timely. If it is pyogenic infection, anti tuberculosis treatment will not obtain the ideal therapeutic effect, and vice versa. So it is important to distinguish them firstly.

\section{Discussion}

The sacroiliac joint accounts for approximately $16 \%$ to $30 \%$ of cases of chronic low back pain, which is often overlooked. Especially young adults, when they complain of pain of the buttock, the reason is often thought to be the lumbar disc herniation, after conventional treatment, the symptoms often did not improve significantly.

In fact, it is difficult to distinguish sacroiliac joint disease from other causes of low back pain based on history alone. Accurate diagnosis is often delayed because of the low incidence of the disease, low clinical suspicion rate, and demonstrating similar symptoms with hip, lumbar and abdominal lesions. Clinical manifestations are often diverse, and symptoms and examination results often indicate more common diseases, such as septic arthritis of the hip, lumbar disc herniation or pelvic abscess. The most likely reason may be that clinicians lack of understanding of the disease, the clinical manifestations are not typical, the infection site is not definite, and imitate the characteristics of hip joint suppurative arthritis, iliac osteoarthritis and lumbar disc herniation ${ }^{[1-3]}$. 
Sacroiliac joint infection is relatively rare, representing $1 \%-2 \%$ of all cases of septic arthritis ${ }^{[4]}$, and is usually associated with multiple predisposing factors, including intravenous drug abuse, immune suppression, pregnancy, trauma and infection elsewhere in the body ${ }^{[5]}$. On the early stage X-Ray and computed tomography(CT) is important to exclude "red flags". With the progress of the disease, CT and Magnetic resonance imaging (MRI) can provide valuable information. Especially MRI clearly demonstrates inflammatory reaction and abscess formation around the sacroiliac joint, which is the best imaging modality for diagnosis. MRI has been proved to be the best tool for early diagnosis of sacroiliac joint infection. However, the infection of sacroiliac joint is initially unsuspected upon hospitalization in $50 \%$ of patients with this eventual diagnosis ${ }^{[5]}$. In fact over $40 \%$ of patients with sacroiliac joint infection, the primary site of infection may never be identified ${ }^{[6]}$.

The diagnosis of sacroiliac joint infection depends on the analysis of comprehensive results, such as physical examination results, laboratory examination, imaging findings, etiological examination and treatment response. Etiological examination is of great significance in the diagnosis of sacroiliac joint infection. Puncture of abscess may get the most accurate diagnosis by biopsy, but the specimen may be contaminated, or the abscess location is not easy to puncture. Sacroiliac joint infection is a rare but important diagnosis because failure to treat can lead to high incidence rate, such as joint instability and systemic spread of infection.

\section{Conclusions}

Sacroiliac joint infection is uncommon in clinical, It's initial symptoms are usually nonspecific, often confused with tuberculosis, septic arthritis of the hip, osteitis of the ilium and lumbar disc herniation which make it difficult to early diagnosis. So when encountered similar situations, the sacroiliac joint infection should be considered, and the pathogen should be identified, in order to treat it correctly as soon as possible.

\section{Declarations}

\section{Ethical approval and informed consent}

This study did not require ethical approval and informed consent as it did not involve patient data directly.

\section{Consent for publication}

Written informed consent for publication was obtained from all participants.

\section{Availability of data and materials}

All data generated or analysed during this study are included in this published article 


\section{Competing interests}

The authors declare that they have no competing interests.

\section{Funding}

This work was supported by Research funds of Hunan Health Commission (No.20201318).

\section{Authors' contributions}

All authors have intellectually contributed to this manuscript. YJL and MY wrote the manuscript. KC and YG analyzed and interpreted the patient data. JPX helped finishing the surgery. KC and MY reviewed the clinical notes and edited the manuscript. All authors read and approved the final manuscript.

\section{Acknowledgements}

Not applicable

\section{References}

1. Dunn EJ, Bryan DM, Nugent JT, et al. Pyogenic infection of the sacroiliac joint. Clin Orthop. 1976;118:113-7.

2. Gordon G, Kabins SA. Pyogenic sacroiliitis. Am J Med. 1980;69:50-6.

3. Osman AA, Govender S. Septic sacroiliitis. Clin Orthop. 1995;313:214-9.

4. Hodgson BF. Pyogenic sacroiliac joint infection. Clin Orthop. 1989;246:146-9.

5. Doita M, Yoshiya S, Nabeshima Y, et al. Acute pyogenic sacroiliitis without predisposing conditions. Spine. 2003;28(18):384-9.

6. Vyskocil JJ, Mcllroy MA, Brennan TA, et al. Pyogenic infection of the sacroiliac joint. Case reports and review of the literature. Medicine. 1991;70:188-97.

\section{Figures}



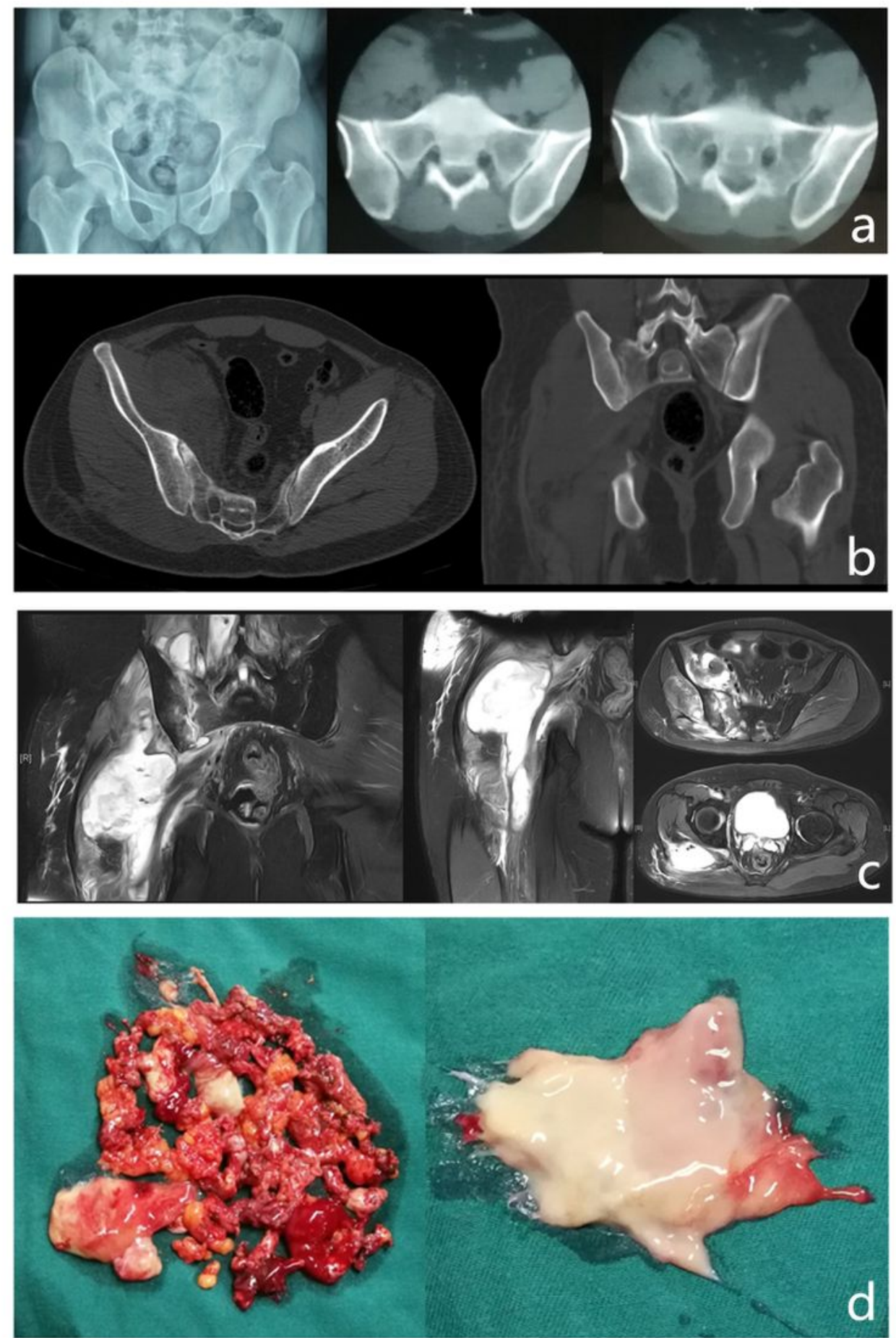

\section{Figure 1}

a: There is no obvious abnormality found in the X-ray and CT. b: The sacroiliac joint space is narrowing, cortical discontinuity of articular face and osteosclerosis. c: MRI manifest that abscess formation and bone marrow edema on the right side of sacral vertebra. $d$ : The infected tissue and abscess. 